7. Bell DM and WHO Working Group on Prevention of International and Community Transmission of SARS. Public health interventions and SARS spread, 2003. Emerg Infect Dis 2004;10:1900-1906.

8. Malone JD, Brigantic R, Muller GA et al. US airport entry screening in response to pandemic influenza: modeling and analysis. Travel Med Infect Dis 2009;7:181-191.
9. Hamilton PA, Marcos LS, Secic M. Performance of infrared ear and forehead thermometers: a comparative study in 205 febrile and afebrile children. J Clin Nurs 2013;22:2509-2518.

10. Geijer H, Udumyan R, Lohse G, Nilsgard Y. Temperature measurements with a temporal scanner: systematic review and meta-analysis. BMJ Open 2016;6:e009509.

\title{
Risk of coronavirus disease 2019 (COVID-19) acquisition among emergency department patients: A retrospective case control study
}

\author{
Jessica P. Ridgway MD, MS ${ }^{1}$ (1) and Ari A. Robicsek MD² \\ ${ }^{1}$ Department of Medicine, University of Chicago, Chicago, Illinois and ${ }^{2}$ Providence St. Joseph Health, Seattle, Washington
}

With the onset of the coronavirus disease 2019 (COVID-19) pandemic, emergency departments (EDs) have seen significant declines in patient volume, partly due to patients' fear of contracting COVID-19 in the ED., ${ }^{1,2}$ Nosocomial transmission of severe acute respiratory coronavirus virus 2 (SARS-CoV-2) has been reported in some healthcare settings, ${ }^{3}$ but little is known about the risk of acquiring COVID-19 in the ED. The objective of this study was to determine whether ED colocation with COVID-19 patients is associated with COVID-19 acquisition.

\section{Methods}

\section{Study design and participants}

We performed a retrospective case control study among patients treated in 39 EDs in the western United States. Patients were included as cases if they visited (and were discharged home from) an ED between March 1, 2020, and July 19, 2020 and subsequently had a positive SARS-CoV-2 PCR test 7-21 days following the ED encounter. Cases were matched with 2 controls who visited (and were discharged from) the same ED within 6 days of the case patient. Controls differed from cases in that they had a negative SARS-CoV-2 PCR test 7-21 days after their ED visit. To ensure that study participants did not have COVID-19 at the time of their ED encounter, we excluded patients who presented to the ED with fever, chills, cough, or shortness of breath. Symptoms were identified using natural language processing of the ED provider notes and chief-complaint documentation. We also excluded patients tested for or diagnosed with COVID-19 during the ED visit.

\section{Data collection and analysis}

For cases and controls, we collected demographic information and the Emergency Severity Index (ESI ${ }^{4}$ from the electronic medical record. To assess exposure to COVID-19 in the ED, we measured the number of COVID-19 patients in the ED in the 24 hours prior to each patient's arrival and the number of minutes each patient

Author for correspondence: Jessica Ridgway, E-mail: Jessica.ridgway@uchospitals.edu Cite this article: Ridgway JP and Robicsek AA. (2021). Risk of coronavirus disease 2019 (COVID-19) acquisition among emergency department patients: A retrospective case control study. Infection Control \& Hospital Epidemiology, 42: 105-107, https://doi.org/ $10.1017 /$ ice.2020.1224 was colocated in the ED with COVID-19 patients. As a proxy for the incidence of COVID-19 in a patient's community, we also measured the percentage of positive tests in the patient's home ZIP code in the 14 days prior to ED visit.

We performed a bivariate analysis comparing characteristics of cases versus controls using the $\chi^{2}$ and the Student $t$ tests, and we used multivariate conditional logistic regression to determine whether ED colocation with COVID-19 patients was associated with case versus control status. This study was approved by the Providence St. Joseph Health Institutional Review Board.

\section{Results}

We identified 102 cases. All cases were matched to 2 controls, except for 3 cases for whom only 1 control could be identified, resulting in 201 controls. In the bivariate analysis, cases were younger (mean age, 46.4 vs 52.2 years; $P=.026$ ), more likely to be Hispanic (39.2\% vs $18.4 \% ; P=.0003)$, more likely to have an ESI of $4-5(31.7 \%$ vs $18.9 \% ; P=.006)$, and more likely to live in a ZIP code with $>14 \%$ COVID- 19 test positivity compared to controls $(47.1 \%$ vs $33.3 \% ; P=.024)$. There was no difference in the bivariate analysis between cases and controls in the number of ED COVID-19 patients or in length of time colocating with COVID-19 patients in the ED (Table 1).

In the multivariate model, patients of Hispanic ethnicity were more likely to acquire COVID-19 compared to whites (aOR, 7.04; 95\% CI, 2.85-17.40), and patients presenting to the ED with an ESI of 4-5 were more likely to acquire COVID-19 than patients with an ESI of 2 (aOR, 3.36; 95\% CI, 1.11-10.22) (Table 1). In the multivariate model, neither time of ED colocation with COVID-19 patients nor number of ED COVID-19 patients was associated with COVID-19 acquisition.

\section{Discussion}

In this retrospective case-control study, we found that ED colocation with COVID-19 patients is not associated with COVID-19 acquisition. Our findings provide reassurance that SARS-CoV-2 transmission occurs uncommonly in EDs. Many EDs have implemented various strategies to limit SARS-CoV-2 transmission, including the use of personal protective equipment such as face masks and eye protection, cohorting patients with respiratory symptoms, social distancing, and limiting visitors. ${ }^{5-7}$ 
Table 1. Comparison of Cases and Controls in Bivariate and Multivariate Analyses

\begin{tabular}{|c|c|c|c|c|}
\hline Characteristic & $\begin{array}{c}\text { Cases }(\mathrm{N}=102), \\
\text { Mean (SD) or No. }(\%)\end{array}$ & $\begin{array}{c}\text { Controls }(\mathrm{N}=201) \\
\text { Mean (SD) or No. }(\%)\end{array}$ & $P$ Value & $\begin{array}{l}\text { aOR in Multivariate } \\
\text { Model }(95 \% \mathrm{Cl})\end{array}$ \\
\hline Age, y (SD) & $46.4(22.7)$ & $52.2(21.1)$ & .026 & $1.00(0.99-1.02)$ \\
\hline \multicolumn{5}{|l|}{ Race/Ethnicity } \\
\hline Black & $4(3.9)$ & $10(5.0)$ & .0003 & $1.58(0.40-6.28)$ \\
\hline Hispanic & 40 (39.2) & $37(18.4)$ & & $7.04(2.85-17.40)$ \\
\hline White & $41(40.2)$ & $128(63.7)$ & & Reference \\
\hline Other/Unknown & $17(16.7)$ & $26(12.9)$ & & $1.95(0.82-4.63)$ \\
\hline Social Vulnerability Index, percentile (SD) & $0.59(0.25)$ & $0.58(0.27)$ & .58 & $1.04(0.31-3.50)$ \\
\hline \multicolumn{5}{|l|}{ Emergency Severity Index } \\
\hline 2 & $8(7.9)$ & $33(16.4)$ & .006 & Reference \\
\hline 3 & $62(61.4)$ & $130(64.7)$ & & $2.25(0.88-5.73)$ \\
\hline $4-5$ & $32(31.7)$ & $38(18.9)$ & & $3.36(1.11-10.22)$ \\
\hline \multicolumn{5}{|l|}{ \% COVID-19 test positivity in home ZIP code } \\
\hline$<2$ & $24(23.5)$ & $46(22.9)$ & & Reference \\
\hline $2-6$ & $17(16.7)$ & $36(17.9)$ & & $0.60(0.20-1.79)$ \\
\hline $7-13$ & $13(12.7)$ & $52(25.9)$ & & $0.27(0.08-0.86)$ \\
\hline $14-19$ & $26(25.5)$ & $27(13.4)$ & 0.024 & $2.00(0.60-6.69)$ \\
\hline$>20$ & $22(21.6)$ & $40(19.9)$ & & $0.86(0.23-3.05)$ \\
\hline \multicolumn{5}{|c|}{ No. patients with COVID-19 in ED in the 24 hours prior to arrival } \\
\hline 0 & $19(18.6)$ & $47(23.4)$ & 0.41 & Reference \\
\hline $1-5$ & $50(49.0)$ & $83(41.3)$ & & $2.49(0.75-8.24)$ \\
\hline$>5$ & $33(32.4)$ & $71(35.3)$ & & $0.83(0.17-4.12)$ \\
\hline Minutes of ED colocation with COVID-19 patients & & & 0.94 & \\
\hline 0 & $36(35.3)$ & $69(34.3)$ & & Reference \\
\hline $1-500$ & $46(45.1)$ & $89(44.3)$ & & $1.08(0.47-2.47)$ \\
\hline$>500$ & $20(19.6)$ & $43(21.4)$ & & $1.32(0.45-3.88)$ \\
\hline
\end{tabular}

Note. SD, standard deviation; ED, emergency department; aOR, adjusted odds ratio; $\mathrm{Cl}$, confidence interval.

The EDs in this study may have implemented different infection control precautions at different times, and we did not seek to determine which strategies are most effective for reducing SARS-CoV-2 transmission.

Since the start of the COVID-19 pandemic, ED patient volume has dropped $41.5 \%-63.5 \%{ }^{1}$ Although some of the reduction in ED volume may be explained by patients with nonemergency conditions avoiding EDs, evidence exists that patients with serious medical emergencies may also be foregoing ED care. ${ }^{2}$ Indeed, ED visits for serious, time-sensitive health conditions like cerebrovascular accidents and myocardial infarctions have significantly declined since the start of the COVID-19 pandemic. ${ }^{8,9}$ This decline in ED volume is likely in part due to fear of contracting COVID-19 in the ED. ${ }^{2}$ Our results suggest that this fear may be unfounded.

Although colocation with COVID-19 patients in the ED was not associated with COVID-19 acquisition in our study, Hispanic patients had higher likelihood of acquiring COVID-19 than white patients. In the United States overall, Hispanic individuals have been disproportionately affected by COVID-19, accounting for 33\% of COVID-19 cases in which the ethnicity of the individual was known, although they make up only $16.7 \%$ of the population. ${ }^{10}$
In summary, in a retrospective case-control study from 39 US EDs, we found that ED colocation with COVID-19 patients was not associated with acquisition of COVID-19. Our findings may provide reassurance that patients who receive care in EDs are not likely at increased risk of contracting COVID-19.

Acknowledgments. We acknowledge Tom French for his contributions to the statistical analysis.

Financial support. No financial support was provided relevant to this article.

Conflicts of interest. All authors report no conflicts of interest relevant to this article.

\section{References}

1. Jeffery MM, D'Onofrio G, Paek H, et al. Trends in emergency department visits and hospital admissions in health care systems in 5 states in the first months of the COVID-19 pandemic in the US. JAMA Intern Med 2020 Aug 3. doi: 10.1001/jamainternmed.2020.3288.

2. Wong L, Hawkins J, Langness S, Murrell K, Iris P, Sammann A. Where are all the patients? addressing COVID-19 fear to encourage sick patients to seek emergency care. NEJM Catalyst 2020. doi: 10.1056/CAT.20.0193. 
3. Rickman HM, Rampling $\mathrm{T}$, Shaw $\mathrm{K}$, et al. Nosocomial transmission of COVID-19: a retrospective study of 66 hospital-acquired cases in a London teaching hospital. Clin Infect Dis 2020 Jun 20. doi: 10.1093/cid/ ciaa816.

4. Emergency Severity Index (ESI): a triage tool for emergency departments. Agency for Healthcare Research and Quality website. https://www.ahrq. gov/professionals/systems/hospital/esi/index.html. Published September 2012. Updated May 2020. Accessed September 22, 2020.

5. Wee LE, Fua TP, Chua YY, et al. Containing COVID-19 in the emergency department: the role of improved case detection and segregation of suspect cases. Acad Emerg Med 2020;27:379-387.

6. Perlini S, Canevari F, Cortesi S, et al. Emergency department and out-of-hospital emergency system (112-AREU 118) integrated response to coronavirus disease 2019 in a Northern Italy centre. Intern
Emerg Med 2020 Jun 8 [Epub ahead of print]. doi: 10.1007/s11739-02002390-4.

7. Stanford KA, Friedman EE, Schmitt J, et al. Routine screening for HIV in an urban emergency department during the COVID-19 pandemic. AIDS Behav 2020;24:2757-2759.

8. Bres Bullrich M, Fridman S, Mandzia JL, et al. COVID-19: stroke admissions, emergency department visits, and prevention clinic referrals. Can J Neurol Sci 2020 May 26. doi: 10.1017/cjn.2020.101.

9. Garcia S, Albaghdadi MS, Meraj PM, et al. Reduction in ST-segment elevation cardiac catheterization laboratory activations in the United States during the COVID-19 pandemic. J Am Coll Cardiol 2020;75:2871-2872.

10. Stokes EK, Zambrano LD, Anderson KN, et al. Coronavirus disease 2019 case surveillance-United States, January 22-May 30, 2020. Morb Mortal Wkly Rep 2020;69:759-765.

\title{
Assessment of room quality of manual cleaning and turnaround times with and without ultraviolet light at an academic medical center
}

\author{
Oluchi J. Abosi MB ChB, MPH, Takaaki Kobayashi MD, Stephanie Holley MBA, BSN, Mary E. Kukla BSN, RN, \\ Angelique Dains BSN, RN, Tatiana Izakovic MD, MHA, Kyle E. Jenn BSN, RN, Holly Meacham RN, MSN, \\ Glen H. Rogers BS, CHESP, Jon Garringer CMIP, William Millard CHESP, CMIP, Daniel J. Diekema MD, MS, \\ Michael B. Edmond MD, MPH, MPA, MBA, Melanie Wellington MD, PhD and Jorge L. Salinas MD \\ University of lowa Hospitals \& Clinics, lowa City, lowa
}

Manual cleaning is the recommended method of environmental disinfection and plays a key role in the prevention of healthcareassociated infections. ${ }^{1}$ Recently, automated "no-touch" disinfection technologies such as ultraviolet (UV) light have been proposed to supplement manual cleaning. ${ }^{2,3}$ In our institution, we implemented UV light for terminal disinfection of contact precautions rooms at discharge. However, we hypothesized that UV light may affect quality of manual cleaning by possibly leading to complacency because housekeepers may feel that it replaces the need for thorough manual cleaning. Furthermore, UV light adds time to the overall cleaning process. We evaluated the impact of adding UV light on the quality of manual cleaning and on room turnaround times.

\section{Methods}

The University of Iowa Hospitals \& Clinics is an 811-bed academic medical center. According to hospital policy, contact precautions rooms undergo manual cleaning with bleach and UV light using continuous UVC waves (Tru-D SmartVUC, PDI Healthcare, Woodcliff Lake, NJ). UV light time is determined automatically based on room size. ${ }^{4}$ Rooms without contact precautions are cleaned using quaternary ammonium only (no UV light). During January-September 2019, we obtained a convenience sample of rooms cleaned at discharge (24 UV-light rooms; 201

Author for correspondence: Oluchi Abosi, E-mail: oabosi@healthcare.uiowa.edu Cite this article: Abosi OJ, et al. (2021). Assessment of room quality of manual cleaning and turnaround times with and without ultraviolet light at an academic medical center. Infection Control \& Hospital Epidemiology, 42: 107-108, https://doi.org/10.1017/ ice.2020.1246
non-UV-light rooms). Each room was assigned a thoroughness of disinfection cleaning (TDC) score of environmental surfaces. ${ }^{5}$ The rooms were identified for TDC assessment after patient admission and were selected randomly by an infection preventionist. Housekeepers were not informed of which rooms were selected. Fluorescent markers, which are not affected by UV light, were applied on high-touch surfaces once discharge orders were placed, and thoroughness was assessed at the completion of terminal cleaning. TDC scores were defined as the percentage of cleaned surfaces out of the total examined surfaces. This information was shared with staff.

We compared TDC scores between UV-light rooms and non-UV-light rooms. We also calculated the manual cleaning time, UV-light cycle durations, and total turnaround time for rooms undergoing terminal disinfection (2,178 UV light rooms and 50,877 non-UV-light rooms). Room turnaround time included time for bed making, room preparation before and after the UV-light procedure, and UV light set-up and dismount, where applicable. No preoccupancy recovery time was needed.

\section{Results}

We assessed 2,383 surfaces in 24 rooms with UV-light disinfection and 201 rooms without UV-light disinfection. The TDC scores were similar in rooms with UV-light disinfection (243 of 273 surfaces) and rooms without UV-light disinfection (1,835 of 2,110 surfaces; $89 \%$ vs $87 \%$ ) (Table 1 ). The housekeepers' median manual cleaning time for the UV-light-treated rooms was 56 minutes (interquartile range [IQR], 37-79 minutes) versus 33 minutes (IQR, 22-43 minutes) for untreated rooms. The 